\title{
RECENSIÓN
}

\section{EPIDAT 3.0 \\ PROGRAMA PARA ANÁLISIS EPIDEMIOLÓGICO DE DATOS TABULADOS}

Xurxo Hervada Vidal, Ma Isolina Santiago Pérez, Enrique Vázquez Fernández, Carlos Castillo Salgado, Enrique Loyola Elizondo y Luis Carlos Silva Ayçaguer.

Editan: Dirección Xeral de Saúde Pública, Xunta de Galicia, Organización Panamericana de la Salud. Año 2003. Recensión: Luis Carlos Silva Ayçaguer. Instituto Superior de Ciencias Médicas de la Habana. La Habana, Cuba.

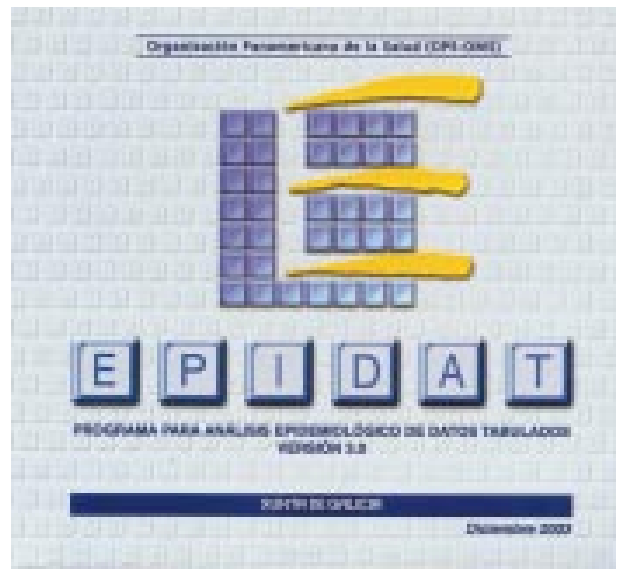

Como para cualquier otro tipo de investigación que opere con datos numéricos, los estudios longitudinales suelen requerir de análisis estadísticos con mayor u menor grado de complejidad. Consecuentemente, es harto frecuente (incluso, inevitable, en la actualidad) que el investigador acuda a recursos computacionales (software) que contribuyan al manejo ágil y correcto de los datos.

Numerosos paquetes informáticos para el tratamiento estadístico y gráfico de datos se pueden hallar en el mercado (EPI2000, SPSS, S-PLUS, SAS, Minitab o STATA, por

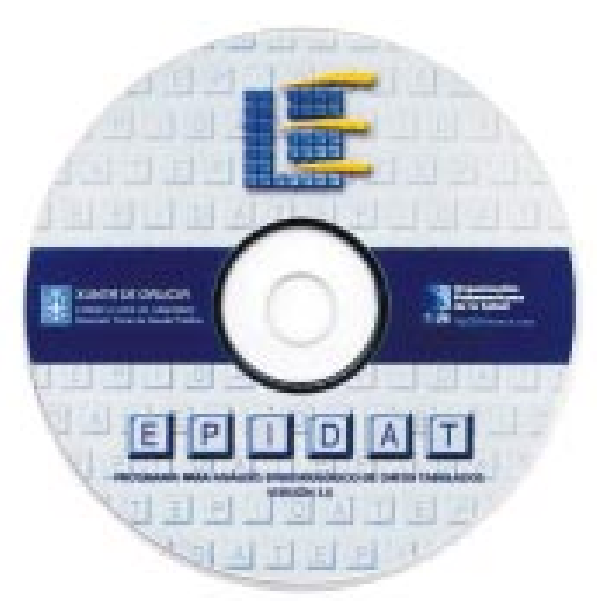

solo mencionar los que acaso sean más conocidos por salubristas y epidemiólogos iberoamericanos). Epidat 3.0, objeto de esta breve reseña, es una opción complementaria a las mencionadas.

Se trata de un programa de libre distribución desarrollado por el Servicio de Información sobre Saúde Pública de la Dirección Xeral de Saúde Pública de la Consellería de Sanidade (Xunta de Galicia) en colaboración con el Área de Análisis de Salud y Sistemas de Información Sanitaria de la Organización Panamericana de la Salud (OPS- 
OMS), y en cuyo equipo de autores se aunaron esfuerzos de especialistas de estas dos entidades y del Instituto Superior de Ciencias Médicas de La Habana (Cuba).

Sus antecedentes se remontan a diciembre de 1994 cuando apareció la Versión 1.0, entonces una calculadora básica diseñada para el entorno MS-DOS. La Versión 2.0, ya en el ambiente Windows, apareció en 1997; y la actual versión, disponible desde 2004, opera en ambiente Windows (98SE o superior).

Epidat 3.0 está concebido como una herramienta para epidemiólogos y otros profesionales de la salud y se orienta a facilitar el manejo de datos ya tabulados.

Este es uno de los elementos distintivos del programa, que contribuye al carácter complementario de EPIDAT respecto de otras aplicaciones: en general, en todos los módulos trabaja con datos agregados, a partir de los cuales se puede realizar una serie de análisis no previstos en las aplicaciones más conocidas (al menos no de una forma tan sencilla y directa). Dicho de otro modo, en lugar de gestionar bases de datos primarios, parte del hecho de que tal gestión puede llevarse adelante de manera muy eficiente con recursos ampliamente disponibles (por ejemplo, Microsoft Excel y Microsoft Access) y opera directamente con bases confeccionadas en dichos entornos, o bien con datos tecleados directamente por el usuario. Consecuentemente, en casi todos los módulos contempla la posibilidad de importar los datos de forma automática a partir de archivos con tablas en formato Dbase, Access o Excel.

El segundo elemento distintivo del programa es que la detallada ayuda en hipertexto de que se le ha dotado, en lugar de poner el énfasis en sus aspectos operativos, ha sido confeccionada con un enfoque a la vez didáctico y crítico, que incluye un amplio abanico de problemas prácticos incorporados en calidad de ejemplos, así como funda- mentos históricos, estadísticos y epidemiológicos de los procedimientos empleados para resolverlos. De ese modo, además de orientarse a incrementar la capacidad analítica de los profesionales de salud interesados en la investigación, Epidat 3.0 procura servir como fuente para la formación autodidacta en materia de estadística aplicada a la epidemiología. Para ello se ha contado con la colaboración ad hoc de un crecido número de especialistas en diferentes áreas temáticas.

Para satisfacer el espíritu de servir como complemento de los programas actualmente disponibles, se ha procurado incorporar técnicas específicas que, siendo de interés actual o potencial de los investigadores, constituyen lagunas en el escenario informático contemporáneo. Para ello se han implementado varios módulos ausentes en las versiones previas (por ejemplo, Demografía, Meta-análisis, Jerarquización y Análisis bayesiano) así como numerosas opciones adicionales dentro de los que ya figuraban en la versión previa. Tal configuración dimana de un sopesado examen de la literatura actual, que se verificó tratando de no comprometer el espíritu abarcador de Epidat 3.0, pero sin aturdir al usuario con propuestas poco demandadas o escasamente promisorias.

Epidat 3.0 ofrece así un amplio espectro de posibilidades analíticas, parte de las cuales podrían ser a nuestro juicio de gran utilidad para quienes lleven adelante estudios longitudinales. Algunos procedimientos incluidos son de índole general y susceptibles de aplicar en virtualmente cualquier estudio en el que las preguntas de investigación lo demanden (cálculo de probabilidades, técnicas para la evaluación de concordancia, índice de desarrollo humano, técnicas de selección muestral o valoración bayesiana de pruebas estadísticas convencionales, por mencionar algunos ejemplos ilustrativos del diapasón presente en el programa); otros, sin embargo, son propios de los estudios longitudinales o 
preferiblemente empleados en dichos estudios, tales como los relacionados con determinación de tamaños muestrales para ese caso, técnicas para evaluación de esperanza de vida, regresión logística para datos agrupados, análisis de tablas de contingencia en el marco exposición-enfermedad, vigilancia epidemiológica y determinadas expresiones del meta-análisis.

EPIDAT 3.0 está disponible gratuitamente en http://dxsp.sergas.es

\section{CONTENIDO GENERAL DE EPIDAT 3.0 SEGÚN MÓDULOS Y SUBMÓDULOS}

\section{Ajuste de tasas}

- Método directo

- Método indirecto

\section{Demografía}

- Pirámides e indicadores demográficos

- Tablas de mortalidad abreviadas

- APVP

- Descomposición del cambio en la esperanza de vida

- Años de esperanza de vida perdidos

\section{Muestreo}

- Cálculo de tamaños de muestra

- Selección muestral

- Asignación de sujetos a tratamientos

\section{Distribuciones de probabilidad}

- Cálculo de probabilidades
- Generación de distribuciones

\section{Concordancia y consistencia}

- Concordancia

- Dos observadores, dos o más categorías

- Tres o más observadores

- Comparación de kappas

- Consistencia

- Alfa de Cronbach

Pruebas diagnósticas

- Pruebas simples

- Pruebas múltiples

- Prueba de referencia imperfecta

- Curvas ROC

- Curva de Lorenz

\section{Tablas de contingencia}

- Exposición-enfermedad

- Tablas $2 \times 2$

- Tablas 2 x N

- Tablas generales

- Tablas M x N

- Regresión logística

\section{Inferencia sobre parámetros}

- Una población

- Dos poblaciones 


\section{Análisis bayesiano}

- Proporción

- Media

- Tablas de contingencia

- Valoración bayesiana de pruebas convencionales

\section{Vigilancia en salud pública}

- Captura-Recaptura

- Detección de clusters

- Gráficos

- Ondas epidémicas

- Efectividad vacunal

\section{Meta-análisis}

- Odds Ratios
- Riesgos relativos

- Diferencia de riesgos

- Diferencia estandarizada de medias

\section{Jerarquización}

- Coeficiente de Gini y curva de Lorenz

- Índice y curva de concentración

- Índice de necesidad en salud

- Índice de desarrollo en salud comunitaria

- Índice de inequidades en salud

- Índice de disimilitud

- Índice de desarrollo humano

- Índice de desarrollo relativo al género 